\title{
Measuring Structural Similarity in Large Online Networks
}

\author{
Abstract \\ Structural similarity based on bipartite graphs can be used to detect meaningful communities, but \\ the networks have been tiny compared to massive online networks. Scalability is important in \\ applications involving tens of millions of individuals with highly skewed degree distributions. \\ Simulation analysis holding underlying similarity constant shows that two widely used measures \\ - Jaccard index and cosine similarity - are biased by the distribution of out-degree in web-scale \\ networks. However, an alternative measure, the Standardized Co-incident Ratio (SCR), is \\ unbiased. We apply SCR to members of Congress, musical artists, and professional sports teams \\ to show how massive co-following on Twitter can be used to map meaningful affiliations among \\ cultural entities, even in the absence of direct connections to one another. Our results show how \\ structural similarity can be used to map cultural alignments and demonstrate the potential \\ usefulness of social media data in the study of culture, politics, and organizations across the \\ social and behavioral sciences.
}

Keywords: Bipartite, Jaccard, cosine similarity, Twitter, co-following

\section{Introduction}

Growing availability of social media data provides unprecedented opportunities to use bipartite graphs to reveal unobserved communities among celebrities, organizations, topics, issues, news items, events, books, bands, and videos (Golder and Macy, 2014). A bipartite graph consists of two modes, such as celebrities and their followers on Twitter, based on the relationships between members of one mode (e.g. followers) and members of the other (e.g. those followed). These 
online relationships might include following, liking, commenting, replying, retweeting, purchasing, listening, or watching. For example, co-following on Twitter can be represented as a bipartite network of followers and the friends they follow. The key behavioral assumption is that the greater the similarity between $i$ and $j$, the higher the probability that a user who follows $i$ will also follow $j$. These similarities and differences among those who are followed can be used to detect meaningful communities, even if the members have no social ties to one another and even if we are unable to measure the attributes of the members. Simply put, instead of classifying the nodes ourselves, based on the analysis of ties and attributes of the nodes, we in effect "crowdsource" the analysis to the millions of users who choose to follow the nodes, based on the assumption that there is meaningful information in those choices that can be extracted using a bipartite graph.

This method need not be limited to communities of people. Bipartite graphs have been used to reduce complex and often ephemeral cultural preferences to simpler and more durable structures (Mohr, 1998). For example, belief graphs have been used to detect cognitive clusters using the associations between individuals and beliefs (Boutyline and Vaisey, forthcoming; Martin, 2002). Bipartite graphs have also been used in content analysis that links words and texts (Tilly, 1997; Baldassarri and Diani, 2007). Book co-purchases on Amazon can be used to differentiate clusters of similar content, and co-listening on last.fm can be used to identify musical genres, without having to rely on expert knowledge or observational data about the particular cultural entities. In short, bipartite network data from online activities can cover an exceedingly wide variety of topics, ranging from politics to music, from hot-button social issues and events to obscure subcultures. 
Bipartite network analysis is not new. What is new is the scale. The digital records of billions of online transactions and communications promise to have a transformative impact on social science. For the past century, quantitative cultural analysis at population scale has relied on opinion surveys. These instruments typically measure subjective and retrospective self-reports based on a set of questions that researchers think are important, not what respondents care about. In contrast, online data are in real time and capture choices that may not be detected in survey responses. For example, consumer preferences involving the expenditure of money may differ from preferences expressed in response to questions in which respondents are minimally invested and about which they feel constrained by perceptions of socially appropriate attitudes. Although surveys can use stratified random samples to generalize results to the underlying population, which is not possible with data from a convenience sample, online data can capture a significant proportion of the entire population, including hard to reach groups such as the very rich and very poor that are susceptible to undercounting in surveys.

Despite the enormous potential applications of bipartite networks to research on public opinion, culture, politics, mass consumption, and social movements, scalable measures of structural similarity have not been developed and validated for large online networks. These networks have properties that are fundamentally different from small networks. Bipartite networks derived from online activities are not only many orders of magnitude larger, they are also highly skewed in the degree distribution (Barabasi and Albert, 1999). In a bipartite network of the kind found in nearly all previous studies, the degree distribution is constrained by the small numbers of nodes. At web scale, these differences in the distribution could be many orders 
of magnitude. The most popular online celebrities and cultural entities attract tens of millions of followers, purchasers, comments, retweets, and likes, while the vast majority of the entities only attract a handful. The skewness of the degree distribution requires a scalable similarity measure that gives unbiased assessment of similarity between extremely high degree nodes and low degree nodes.

This paper examines whether measures of structural similarity that have been widely used for relatively small offline networks can be applied at Web scale. We use simulation experiments to test the scalability of two widely used continuous measures of structural similarity in bipartite graphs - the Jaccard index (Jaccard, 1901) and cosine similarity. Unlike observational data from empirical networks, simulation experiments provide "ground truth" about the similarity of nodes in networks of different size and structure. Holding similarity constant, we varied the size and structure of the simulated network in order to test whether these measures of similarity are biased in ways that could compromise their use with massively large online networks. The results show that Jaccard index and cosine similarity are biased by the highly skewed degree distributions encountered in large networks. We diagnose the source of the bias and propose an alternative measure, the standardized co-incident ratio (SCR) that avoids the problem by comparing the observed co-follower count to the co-follower count expected in an otherwise identical randomized network. Simulation experiments show that SCR is an unbiased measure.

The second part of the paper applies SCR to data for millions of Twitter co-followers to illustrate the usefulness for discovering meaningful communities in large networks (Garimella and Weber, 2014). Observational data cannot be used to assess the external validity of any of 
structural similarity measures since we have no way to know what attributes attracted the cofollowers or how similar are co-followed users on the relevant attributes. Simply put, with observational data, there is no ground truth similarity with which to assess the similarity predicted by alternative structural measures.

Instead, we used observational data to see if meaningful communities could be detected by the one-mode projection of bipartite graphs when applied across several socio-cultural domains, including politics, music, and sports. We analyzed political co-following using a network of 4.9M followers of the US Congress, 58M followers of U.S. music artists, and 11M followers of professional sports teams. These three co-follower graphs have three important properties:

- Members vary widely in the number of followers and co-followers.

- Members are likely to share the same followers because they share an attribute that is relevant to their followers.

- Members have observable cultural attributes that can be used to measure similarities on multiple dimensions and to see how closely these match with the similarities predicted by structural similarity.

In each application, the communities in the projection of an affiliation network corresponded to those that could be expected: Congressional parties, musical genres, and sports leagues.

\section{Measures and Methods}

Jaccard index and cosine similarity are two widely used continuous measures of structural 
similarity. We use simulation experiments to see if these measures give robust results as network size increases by several orders of magnitude. To preview the results, we find that neither measure is affected by network size, holding the degree distribution constant. However, both measures are affected by differences in degree distribution, holding network size constant. We then show that the bias can be corrected by measuring observed similarity relative to the similarity expected in an otherwise identical randomized network. Remarkably, when measured relative to their expected values, the standardized versions of Jaccard index and cosine similarity converge to the ratio of observed to expected common neighbors, which we refer to as the standardized co-incident ratio (SCR).

\subsection{Measures of Structural Similarity}

Table 1 is used to illustrate alternative measures of structural similarity between nodes $i$ and $j$ in a bipartite network:

Table 1: Cell counts of binary arcs in a bipartite network for constructing continuous pairwise measures of structural similarity.

\begin{tabular}{rl|c|}
\multicolumn{1}{c}{} & Not adjacent to $j$ & Adjacent to $j$ \\
\cline { 2 - 3 } Not adjacent to $i$ & $a$ & $b$ \\
\cline { 2 - 3 } Adjacent to $i$ & $c$ & $d$ \\
\cline { 2 - 3 } & &
\end{tabular}

Standardized co-incident ratio $\left(S C R_{i j}\right)$ measures the similarity of $i$ and $j$ as the ratio of their observed common neighbors ( $d$ in Table 1$)$ and the number expected in an otherwise identical 
randomized network:

$$
S C R_{i j}=\frac{d}{E(d)}
$$

Cosine similarity $\left(C_{i j}\right)$ measures the cosine of the angle between two vectors. For binary vectors, the cosine is the ratio of the common neighbors $(d)$ to the geometric mean of the neighbors of $i$ (or $c+d$ in Table 1) and the neighbors of $j($ or $b+d)$ :

$$
C_{i j}=\frac{d}{\sqrt{(c+d)(b+d)}}
$$

Jaccard index $\left(J_{i j}\right)$ measures structural similarity as the proportion of the number of common neighbors that are neighbors of both. As with cosine similarity, the common neighbors are the numerator, which is standardized by the union of the followers instead of the geometric mean:

$$
J_{i j}=\frac{d}{b+c+d}
$$

Phi Coeffiecient $\left(\phi_{i j}\right)$ is a reduced form of the Pearson product-moment correlation for binary vectors. It is unique in giving equal weight to those who follow neither $i$ nor $j(a)$ as to those who follow both $(d)$. Similarity is then reduced by the number who follow one but not the other, which allows the similarity measure to be negative, a second unique property:

$$
\phi i j=\frac{(a \times d)-(b \times c)}{\sqrt{(a+b) \times(c+d) \times(a+c) \times(b+d)}}
$$

Phi is related to the chi-squared statistic for a $2 \times 2$ contingency table: $\phi^{2}=\frac{\chi^{2}}{\mathrm{~N}}$. Expanding the chi-square, we get 


$$
\phi i j^{2}=\frac{1}{N} \sum_{\mathrm{i}} \sum_{\mathrm{j}} \frac{\left(O_{i j}-E_{i j}\right)^{2}}{E_{i j}}
$$

where $i$ and $j$ are the row and column indices in the $2 \times 2$ matrix, and $O_{i j}$ and $E_{i j}$ are the observed and the expected counts in the cell $i j . N$ is the matrix sum, where the observed count is influenced by the co-absence count $a$ which can be very large at Web scale due to the fact that most nodes attract connections from only a tiny fraction of the population in the network, hence $a$ is likely to be very large relative to $d$. For example, in the Congressional co-following network discussed below, the mean value of $a$ among 552 members is 4,889,037, which is 36,622 times as large as the mean value of $d(133.5)$. In short, the diagonal count swamps the off-diagonal in this network, as is likely in all large-scale online networks. With $a \gg[b, c]$ and $a \times d \gg b \times c^{1}$, the $b \times c$ term in phi's numerator becomes negligible, leaving $a \times d$ as the numerator. In the denominator, $a \gg[b, c]$ means $(a+b) \times(a+c)$ is reduced to $a \times a$. Canceling $a$ in both denominator and numerator then reduces phi to cosine similarity. In short, phi converges with cosine as network size increases. Since our interest is focused on scalability to large networks, we omit consideration of phi and focus on Jaccard index and cosine coefficient, the two most widely used measures of structural similarity.

\subsection{Testing Scalability with Simulation Experiments}

We assess the robustness of Jaccard index and cosine similarity in three experiments. In experiment 1 , we vary network size while holding degree distribution constant. In experiments 2

\footnotetext{
${ }^{1}$ In the Congressional co-follower graph, the mean value of $a$ among all possible pair of Congress members is 198 times as large as the mean value of $b$ or $c$. The mean value of $a \times d$ is 120 times as large as $b \times c$.
} 
and 3, we vary the in-degree and out-degree distribution ${ }^{2}$ while holding network size constant. The experiments use an empirical network drawn from the same Twitter data we use later in the paper to illustrate empirical applications of the similarity measures. The data consists of a set of Twitter accounts of 115 current and recent members of the U.S. Senate and about 1M unique followers.

In experiment 1 , we measure structural similarity as we vary the size of the Senatefollower network by randomly sampling followers. The sampled networks differ in size by orders of magnitude, from 1000 to $1 \mathrm{M}$ but not in network structure. In addition to Jaccard index and cosine similarity, we also calculated a standardized version of the co-follower count which we refer to as the standardized co-incident ratio (or SCR). We have no way to know the latent similarity that is captured by co-follower relations, but random sampling insures that there should only be random variation as network size increases. Hence an unbiased measure of structural similarity should not be sensitive to the size of the network. That is what we find for all three measures. Each measure is mean-centered and normed by the standard deviation to provide a common metric. Figure 1 reports the mean pairwise structural similarity in each network which is invariant over very large differences in network size.

[Figure 1 about here]

However, unlike our experiment, network structure does not remain constant as empirical networks increase in size. The upper bound of degree increases with size while the lower bound does not. We therefore need to also test the robustness of similarity measures to differences in the distribution of degree. Experiment 2 is similar to experiment 1 in that we compare networks

\footnotetext{
${ }^{2}$ In-degree refers to the number of users who follow a Senator and out-degree refers to the number of Senators followed by a user.
} 
created by randomly sampling the nodes, but in this study, we vary the degree distribution while holding the size constant. First, we removed high-degree followers, leaving a subset of followers with similar in-degree. Second, we ranked the Senators by in-degree from the followers who remained and randomly removed 50\% from among the $X \%$ with the highest degree, where $X=[50$, $55,60,75,100]$, such that the Gini coefficient ranges from .3 to .8 . This procedure holds the distribution of out-degree constant as we manipulate in-degree. Figure 2 compares the mean pairwise similarity across networks of identical size whose degree distributions become increasingly skewed as measured by the Gini coefficient. The results show that both the Jaccard index and cosine similarity are unbiased by changes in the distribution of out-degree.

[Figure 2 about here]

Experiment 3 is identical to experiment 2 except that we hold the distribution of indegree constant as we vary out-degree. First, we removed high-degree Senators, leaving a subset of 66 Senators with similar in-degree. Second, we ranked the followers of the remaining Senators by out-degree and removed $25 \%(14,600)$ from among the $X \%$ with the highest degree, where $X=[25,27.5,30,37.5,50,75,100]$, such that the Gini coefficient ranges from .1 to .5. This procedure controls for the possibility that users who follow few Senators prefer those that are most popular. However, it is still possible that those who follow many Senators are less discriminating (Adamic and Adar, 2003). Thus, as we remove followers with high out-degree, we should expect to observe either no change in mean pairwise similarity or an increase in similarity as the less discriminating followers with high out-degree are removed and the degree distribution becomes less skewed. Surprisingly, Figure 3 shows the opposite: Both Jaccard index and cosine similarity are higher when the Gini coefficient is relatively large, that is, when the 
distribution of out-degree is more highly skewed. The reason is straightforward: When the outdegree distribution is highly skewed, there are more high-degree followers who follow almost every Senator. Because of the prevalence of co-following produced by these high-degree followers, $d$ tends to be larger relative to the increase in $a$ and $c$, as defined in Table 1 . That is why we observe an increase in Jaccard index and cosine similarity as Gini increases.

[Figure 3 about here]

In contrast, similarity measured by SCR shows no upward trend. SCR has the same numerator as the other two measures - the number of co-followers of each Senate pair ( $d$ in Table 1). The difference is the denominator. Instead of comparing the number of co-followers with the number of followers (measured either as the union or the geometric mean), SCR controls for the expected increase in co-following among high-degree followers by taking as a baseline the number of co-followers expected by chance in an otherwise identical network. The randomization procedure preserves the original in-degree and out-degree of every node (Zweig and Kauffman 2011; Gionis, Mielikainen, and Tsaparas 2007; Neal 2014). For a randomly selected Senator $S$ with in-degree $k_{S}$ we sample the $k_{S}$ nodes from the set of Senate followers based on a probability that is proportional to the followers' degrees. We assign these followers to $S$ and update the out-degree of the followers. We repeat the procedure until no followers remain to be assigned. ${ }^{3}$ The results in Figure 3 show that the expected similarity in a randomized network effectively reduces the bias introduced by large numbers of followers with high outdegree. High-degree followers are also more likely to co-follow in the randomized network, which cancels out the higher observed co-follower count.

\footnotetext{
${ }^{3}$ The last edge to be shuffled is susceptible to being dropped if there is no way to include the edge without altering the degree of the other node. This problem does not arise with any edges prior to the last.
} 
As additional confirmation, we also standardized the Jaccard index and cosine similarity by their expected values using the same network randomization procedure as for SCR. We found that both measures converge to SCR when the bias introduced by the inflated co-follower count is removed. (The correlation with SCR is 1.0 for the standardized Jaccard index and .99 for the standardized cosine.) For the remainder of the paper we therefore limit the analyses to SCR, the simplest of the three standardized measures.

\section{Empirical Results}

Using the Twitter Public Application Programming Interface (API), we collected three datasets containing all the followers of 552 U.S. Congress members from the $111^{\text {th }}, 112^{\text {th }}$ and $113^{\text {th }}$ Congress, 1368 U.S. music artists, and 123 teams from the five major North American sports leagues. We chose these three domains because the entities are widely followed on Twitter and have in common at least one highly salient and identifiable attribute - party membership, musical genre, and league. Nevertheless, in contrast to the simulation experiments, these attributes cannot be used to directly compare structural and cultural similarity because we have no way to know what attributes attracted the attention of followers, or if the same attributes were equally relevant to all followers. For example, we found that co-following in all three domains reflects status similarity, due to the tendency for people to follow publicly visible celebrities. As a consequence, two celebrities might be co-followed even though they have no other attribute in common except their celebrity. In addition, co-following of politicians and sports teams reflects spatial proximity (e.g. a Senator and Representative might be co-followed by residents of the same Congressional district, and a baseball and football team might be co-followed by fans in the 
same city.) Religion, gender, age, ethnicity, and physical attraction may also have an effect, although we do not have data with which to explore these possible influences.

Although we cannot use empirical observations to verify structural measures of similarity, co-following can be used to see if we can detect hidden community structure (the extent of clustering in the unipartite projection of the network) and to see if these communities are meaningful (i.e., do they correspond with observable attributes that might be expected to differentiate the nodes). If so, co-following provides a potentially useful way to reveal hidden affiliations among widely followed Twitter users, even in the absence of information about their attributes or the relationships among them.

We looked for community structure based on the modularity index (Q) commonly used in the community detection literature (Newman and Girvan, 2004). Modularity measures the extent to which individual nodes are connected with others who share the same community membership. In mathematical form, the modularity of the weighted network can be represented as (Newman, 2004):

$$
Q=\frac{1}{2 w} \sum_{i} \sum_{j}\left(w_{i j}-\frac{w_{i} w_{j}}{2 w}\right) \delta\left(C_{i}, C_{j}\right)
$$

where the edge weight $w_{i j}$ is the structural similarity between two Twitter users $i$ and $j, 2 w$ is the sum of all edge weights in the graph

$$
2 w=\Sigma_{i j}\left(w_{i j}\right),
$$

$w_{i}$ is the sum of all edge weights attached to $i$

$$
w_{i}=\Sigma_{j}\left(w_{i j}\right),
$$


and $C_{i}$ indicates $i$ 's community membership.

Community memberships were detected using Louvain Modularity (Blondel et al. 2008) that finds the partitions that optimize $Q$. The Kronecker delta function $\delta\left(C_{i}, C_{j}\right)$ takes the value 1 if nodes $i$ and $j$ are in the same community and 0 otherwise. The modularity $Q$ of a network could range from -0.5 to 1 , where $Q=0$ means that communities cannot be detected (i.e. each "community" may be nothing more than random clustering); $Q>0$ means that the sum of the weighted edges within the community exceeds the sum expected in the randomized network.

We assess qualitatively whether the communities are meaningful using network visualizations of the unipartite projections, where pairwise node proximities correspond to structural similarity and nodes are colored based on political party, musical genre, and sports league. The qualitative assessments are accompanied as well by a quantitative measure of the $Q$ modularity when $C$ is assigned based on node attributes.

\subsection{Empirical Applications to Politics, Music, and Sports}

Using SRC to measure structural similarity, $Q$ modularity for the unipartite projections of the biparatite co-follower networks for Congress members (.1), musical artists (.349), and North American sports teams (.45) are all positive, indicating that there is a non-random optimal partitioning. The results show that co-following can be used to detect hidden community structure in the affiliations among Congress members, musicians, and sports teams.

Having found non-random community structure in all three co-follower networks, we turn to the more important question as to whether these detected communities are substantively meaningful. Put differently, it is not enough to show that the community structure is non-random, we also want to know if the structure reflects intuitively plausible user attributes that might be 
expected to attract social media followers. To that end, we examine each of the three co-follower networks in turn.

\subsection{U.S. Congress}

Figure $4^{4}$ visualizes the unipartite projected affiliations among 552 widely followed Congress members, where pairwise proximities correspond to structural similarity using SCR, size indicates the relative follower count, and nodes are colored according to party membership as Republican (red), Democrat (blue), or Independent (green). It is immediately evident that Congressional co-following can be used to recover party membership, which suggests we can meaningfully identify the location of individual members in the network of party affiliations derived from structural similarities. Notice that Independents are on the periphery of the blue cluster, with Bernie Sanders most closely affiliated with Democrats who are farthest from Republicans.

As additional support, the $Q$ modularity based on party membership is $Q=.093$, compared to $Q=.1$ using partitions that optimize $Q$. In other words, party membership is very close to the optimal partitioning. These results are consistent with a related study by Bond and Messing (2015) that used Facebook "likes" of political pages to estimate the ideological alignment of politicians and their supporters. Although they use factorization rather than modularity, they find a strong correlation (.94) between their structural measure of ideology and Poole and Rosenthal's (1991; 2001) DW-Nominate measure based on roll call voting.

\footnotetext{
${ }^{4}$ The network visualization follows the procedure introduced in Serrano, Boguñá, and Vespignani (2009) for extracting the "backbone" in complex weighted networks, arcs are deleted if the weight is less than one standard deviations above the mean in the distribution of weights in each node's ego networks. The resulting network is directed. Then the tie weights are mean centered by dividing the mean of the weights in the ego's network. This step is only for keeping the network visually compact while it does not change the relative positions of nodes.
} 
The partisan polarization evident in Figure 4 also speaks to the debate among political scientists as to whether the divisions among political elites reflect divisions within the electorate (Fiorina, Abrams and Pope, 2005; DellaPosta, Shi and Macy, 2015; Abramowitz, 2010; DiMaggio, Evans, and Bryson, 1996; Baldassarri and Gelman, 2008). The polarized affiliations in the network visualization are the one-mode projection of the polarized political preferences among millions of citizens in choosing which members of Congress to follow (see also Bond and Messing, 2015, who reach a similar conclusion).

[Figure 4 about here]

Finally, Figure 4 reveals a tendency for the most popular members to be clustered together on the periphery of the network. This indicates that co-following reflects in part degree similarity, i.e., a tendency of those who follow celebrities to a) follow other celebrities and b) only follow other celebrities. For example, we found that the higher a given politician's follower count, the smaller the number of other politicians with whom that member is co-followed. Additional analyses show that SRC is about equally correlated with similar popularity $(r=.26)$ and with similarity in voting record ( $r=.25)$ as measured by Poole and Rosenthal (2001).

In addition to party membership and popularity, co-follower ties can also be expected to reflect spatial proximity since that is the basis for Congressional representation. Given the "big sort" documented by Bishop (2008), spatial co-following is likely to reinforce partisan affiliation. Table 2 reports the mean pairwise co-follower similarity as measured by SCR, broken down by same-party and between-party pairings.

Table 2. Pairwise SCR Broken Down by State and Party Co-location 


\begin{tabular}{|l|l|l|}
\hline & Same Party & Different Party \\
\hline Same State & 0.16 & 0.09 \\
\hline Different State & 0.10 & 0.06 \\
\hline
\end{tabular}

In addition to party membership and popularity, co-follower ties can also be expected to reflect spatial proximity since that is the basis for Congressional representation. Given the "big sort" documented by Bishop (2008), spatial co-following is likely to reinforce partisan affiliation. Table 2 reports the mean pairwise structural similarity among same-state and between-state Congressional pairings, broken down by same-party and between-party pairings. As expected, people tend to co-follow politicians from the same state, but co-following by geolocation is much weaker than by party affiliation.

\subsection{U.S. Musical Artists}

Using the Rovi $\mathrm{API}^{5}$, we collected genre information for every U.S. musical artist with a Twitter account listed on Musicbrainz.com, a publicly created open content music database. We then used the Twitter public API to obtain the 58M followers of the 1368 artists with at least 10,000 followers in the six most popular genres. Figure 5 is a network visualization in which proximities correspond to pairwise SCR similarities, circle sizes indicate follower counts, and nodes are colored according to genre: Pop/Rock (purple), Rap (green), R\&B (black), Electronic (orange), and Country (red). Music genre produces a high level of modularity $(\mathrm{Q}=0.349)$ as evident in the network visualization. Country and Rap are farthest apart, with R\&B closer to Rap and Pop/Rock closer to Country. The two most cohesive

\footnotetext{
${ }^{5} \mathrm{http}: / /$ developer.rovicorp.com/io-docs
} 
communities are Electronic and Country, while the largest communities, Pop/Rock and Rap, are least cohesive.

[Figure 5 about here]

\subsection{North American Professional Sports Teams}

We collected 11M unique Twitter followers of 123 North American teams with more than 10,000 followers. Figure 6 displays the network visualization, with nodes colored by membership in the five major professional leagues, NFL (green), MLB (blue), NHL (orange), NBA (purple) and MLS (red). The league-based community structure is highly differentiated, as confirmed by much higher modularity $(\mathrm{Q}=.452)$ than found in politics and music. While this may seem obvious after the fact (Watts, 2011), it is also highly plausible that teams might have been clustered by city since that is the common denominator of the fans of professional sports, while other members of the same league are presumably regarded as rivals by devoted fans. While spatial proximity is by far the best predictor of between-league co-follower affinities, the league remains a far stronger organizing principle of the community structure. Soccer is least popular and the least densely connected to other sports, while basketball, football, and baseball are most popular. However, basketball is similar to soccer in its relative isolation from other sports, leaving baseball and football as the "sister leagues."

[Figure 6 about here] 


\section{Discussion and Conclusion}

Analysis of the Congressional co-follower network shows that popularity as well political ideology attracts followers. As a consequence, co-following on Twitter exhibits the bandwagon effect of "preferential attachment." Following a celebrity on Twitter is not only motivated by interest in the attributes of the celebrity but also because everyone else is following that person. This "rich getting richer" effect produces two problems for conventional similarity measures that is designed for small networks. On the mode of those followed, two celebrities could have enormously large numbers of co-followers even though they have nothing in common other than their celebrity.

On the mode of those following, the more people they follow, the less discriminating they are in their choices, which means that they provide less information in evaluating similarity compared to those who follow selectively. In short, differences in degree pose a challenge to measures of structural similarity: how can we know whether co-following indicates a meaningful cultural affiliation that is not contaminated by a celebrity's popularity or their followers' indiscriminate selection? The answer, we propose, is to take into account the number of cofollowers that would be expected by chance, given the distribution of degree. Two widely used measures of structural similarity, Jaccard index and cosine similarity, take into account the number of people that are followed but not the expected number of co-followers. An alternative measure, the standardized co-incident ratio, minimizes the risk of bias due to a highly skewed distribution of degree. 
Applications of SCR to co-followers of politicians, musical artists, and sports teams show how social media data can be used to map cultural alignments across any domain that attracts large numbers of followers. This method does not require access to data about the distribution of cultural attributes or organizational affiliations, nor does it require the existence of, or data about, the social ties among the users that are followed. Where these data are available, modularity analysis can then be used to identify the attributes and/or relationships that map most closely with the pattern of affiliations. Where these data are not available, the affiliations can be used to make inferences about the salient dimensions of cultural alignments.

Bipartite graphs have been long used in social science research to find patterns of associations among culture objects, beliefs and attitudes, and organizational affiliations (Mohr, 1998; Martin, 2002). Online social media open up vast untapped opportunities to apply these same methods to networks that are many orders of magnitude larger than the hand-coded ethnographic data of an earlier era. Indeed, this method is not limited to co-following on Twitter or to the clustering of individuals. It can be used to map the affiliations among books that are copurchased on Amazon, songs that are downloaded on last.fm, and social movement hashtags that are diffused over blogs. The possibilities are limited only by our imagination, our access to online data, and the robustness of our models and measures. We hope to have contributed to advances in the latter.

Acknowledgement: The authors thank Kai Mast, Emma Bell, and Yu-Ting Hsu for their assistance in data collection. The research was supported by the U.S. National Science 
Foundation through SES-1303533, SES-1357488, and SES 1226483, by the Minerva Initiative, and by the National Research Foundation of Korea NRF-2013S1A3A2055285.

\section{References}

Abramowitz, AI., 2010. The disappearing center: Engaged citizens, polarization, and American democracy. New Haven: Yale University Press.

Adamic, Lada A., Eytan Adar, 2003. "Friends and neighbors on the Web." Social Networks 25:211-230.

Barabasi, Albert-Laszlo, Reka Albert, 1999. "Emergence of Scaling in Random Networks." Science 285:509-512.

Bishop, Bill, 2008. The Big Sort: Why the Clustering of Like-Minded America Is Tearing Us Apart. Boston: Houghton Mifflin.

Bond, Robert, Solomon Messing, 2015. "Quantifying Social Media's Political Space: Estimating Ideology from Publicly Revealed Preferences on Facebook." American Political Science Review 109:62-78.

Baldassarri, Delia, Mario Diani, 2007. "The Integrative Power of Civic Networks." American Journal of Sociology 113(3): 735-80.

Baldassarri, D., A. Gelman, 2008. "Partisans Without Constraint: Political Polarization and Trends in American Public Opinion.” American Journal of Sociology, 114: 408-46.

Blondel, Vincent D., Jean-Loup Guillaume, Renaud Lambiotte, Etienne Lefebvre, 2008. "Fast unfolding of communities in large networks." Journal of Statistical Mechanics: Theory and Experiment (10), P10008 (12pp) doi: 10.1088/1742-5468/2008/10/P10008. 
Boutyline, Andrei, Stephen Vaisey, Forthcoming. "Belief Network Analysis: A Relational Approach to Understanding the Structure of Attitudes." American Journal of Sociology.

DellaPosta, Daniel, Yongren Shi, Michael W. Macy, 2015. "Why Do Liberals Drink Lattes?” American Journal of Sociology 120(5):1473-1511.

DiMaggio, P., J. Evans, B. Bryson, 1996. "Have American's Social Attitudes Become More Polarized?" American Journal of Sociology 102:690-755.

Garimella, Venkata Rama Kiran, Ingmar Weber, 2014. “Co-Following on Twitter.” HT '14 Proceedings of the 25th ACM conference on Hypertext and social media: 249-254.

Gionis A, Mannila H, Mielikainen T, Tsaparas P, 2007 Assessing Data Mining Results Via Swap Randomization. ACM Trans. Knowl. Discov. Data 1(3): 14.

Golder, Scott A., Michael W. Macy, 2014. "Digital Footprints: Opportunities and Challenges for Online Social Research.” Annual Review of Sociology 40: 129-152.

Fiorina, Morris P., Samuel J Abrams, Jeremy Pope, 2005. Culture war? New York: Pearson Longman.

Jaccard, Paul, 1901. "Étude comparative de la distribution florale dans une portion des Alpes et des Jura", Bulletin de la Société Vaudoise des Sciences Naturelles 37: 547-579.

Martin, John Levi, 2002. "Power, Authority, and the Constraint of Belief Systems." American Journal of Sociology 107: 861-904.

Mohr, John W., 1998. "Measuring Meaning Structures." Annual Review of Sociology 24:345-70.

Neal, Zachary, 2014. "The backbone of bipartite projections: Inferring relationships from coauthorship, co-sponsorship, co-attendance and other co-behaviors." Social Networks 39:84-97.

Newman, M. E. J., 2004. “Analysis of Weighted Networks.” Phys. Rev. E 70, 056131. 
Newman, M. E. J. and M. Girvan, 2004. "Finding and evaluating community structure in networks." Phys. Rev. E 69, 026113.

Poole, Keith T. and Howard Rosenthal, 1991. "Patterns of Congressional Voting." American Journal of Political Science. 35(1): 228-278.

Poole, Keith T. and Howard Rosenthal, 2001. Congress: A Political-Economic History of Roll Call Voting. Oxford University Press.

Serrano, M. A., M. Boguñá, and A. Vespignani, 2009. "Extracting the multiscale backbone of complex weighted networks". Proceedings of the National Academy of Sciences USA 106, 6438.

Tilly, Charles, 1997. "Parliamentarization of popular contention in Great Britain, 1758-1834." Theory and Society 26(2-3):245-73.Zweig, Katharina A. and Michael Kauffman. 2011. "A systematic approach to the one-mode projection of bipartite graphs" Social Network Analysis and Mining 1:187-218.

Watts, Duncan J., 2011. Everything Is Obvious: Once You Know the Answer. Crown Business. 
Figure 1: Structural similarity measured by SCR, Jaccard index and cosine similarity is unaffected by changes in network size, holding degree distribution constant. We varied network size by randomly sampling the followers of 115 current and recent U.S. Senators who are widely followed on Twitter.

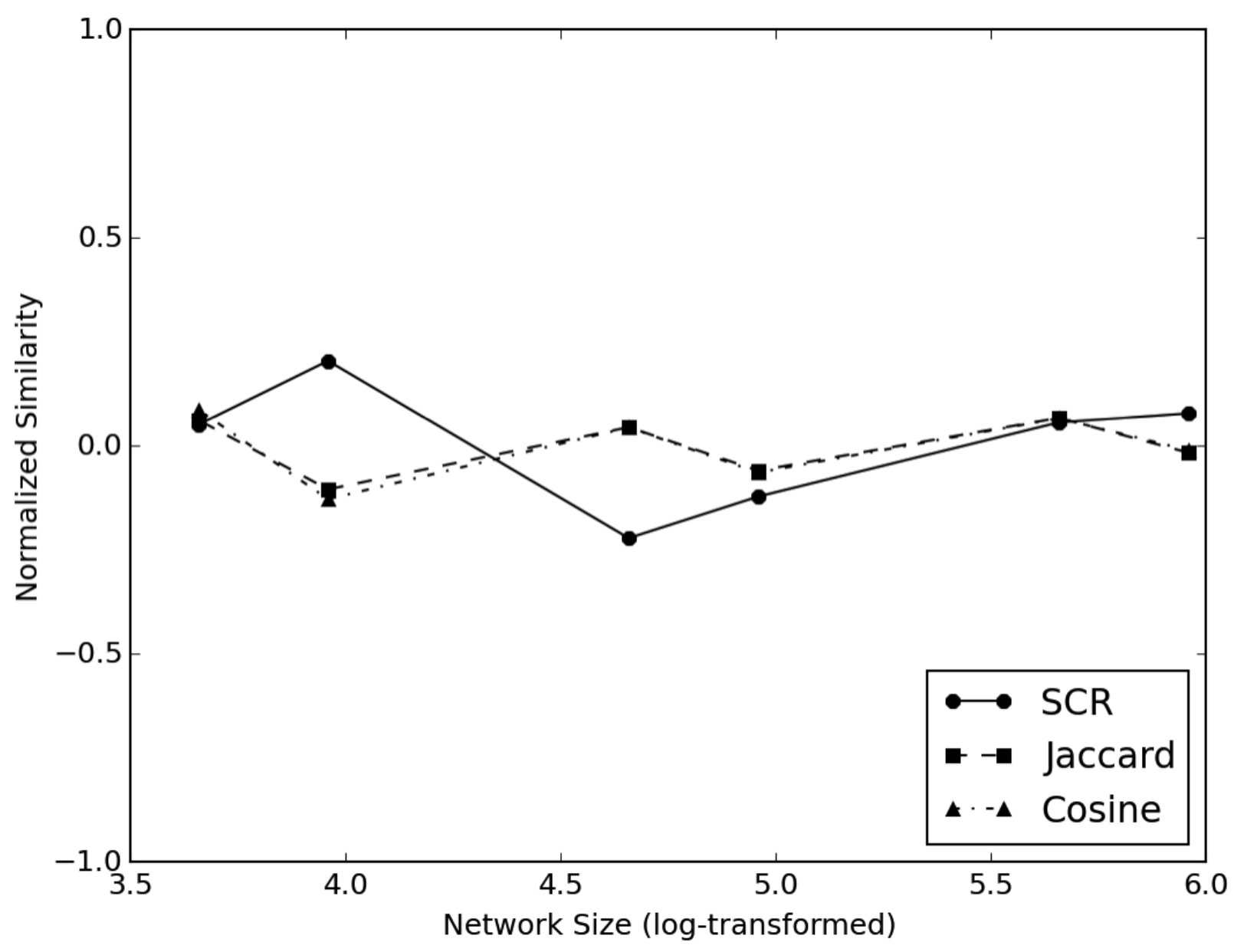


Figure 2: Structural similarity measured by SCR, Jaccard index and cosine similarity is unaffected by changes in the in-degree distribution, holding network size and the outdegree distribution constant. We varied the in-degree distribution by removing $50 \%$ from among the $X \%$ with the highest degree, where $X=[50,55,60,75,100]$, such that the Gini coefficient ranges from .3 to .8 .

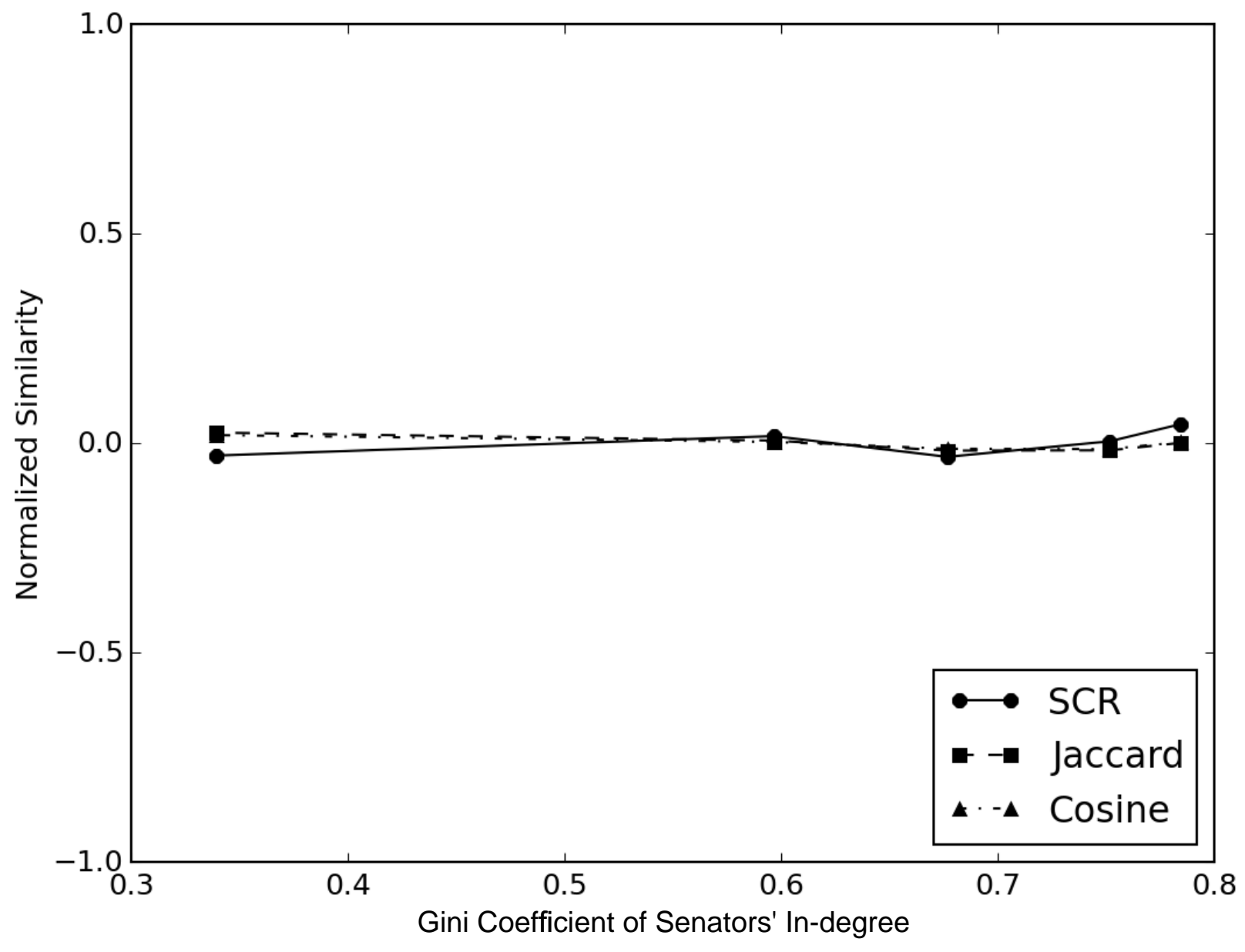


Figure 3: Structural similarity measured by SCR, Jaccard index and cosine similarity is biased by changes in the out-degree distribution, holding network size and the in-degree distribution constant. The design is identical to that in Figure 2 except that we remove high-degree Senators instead of followers, such that the Gini coefficient ranges from .1 to .5.

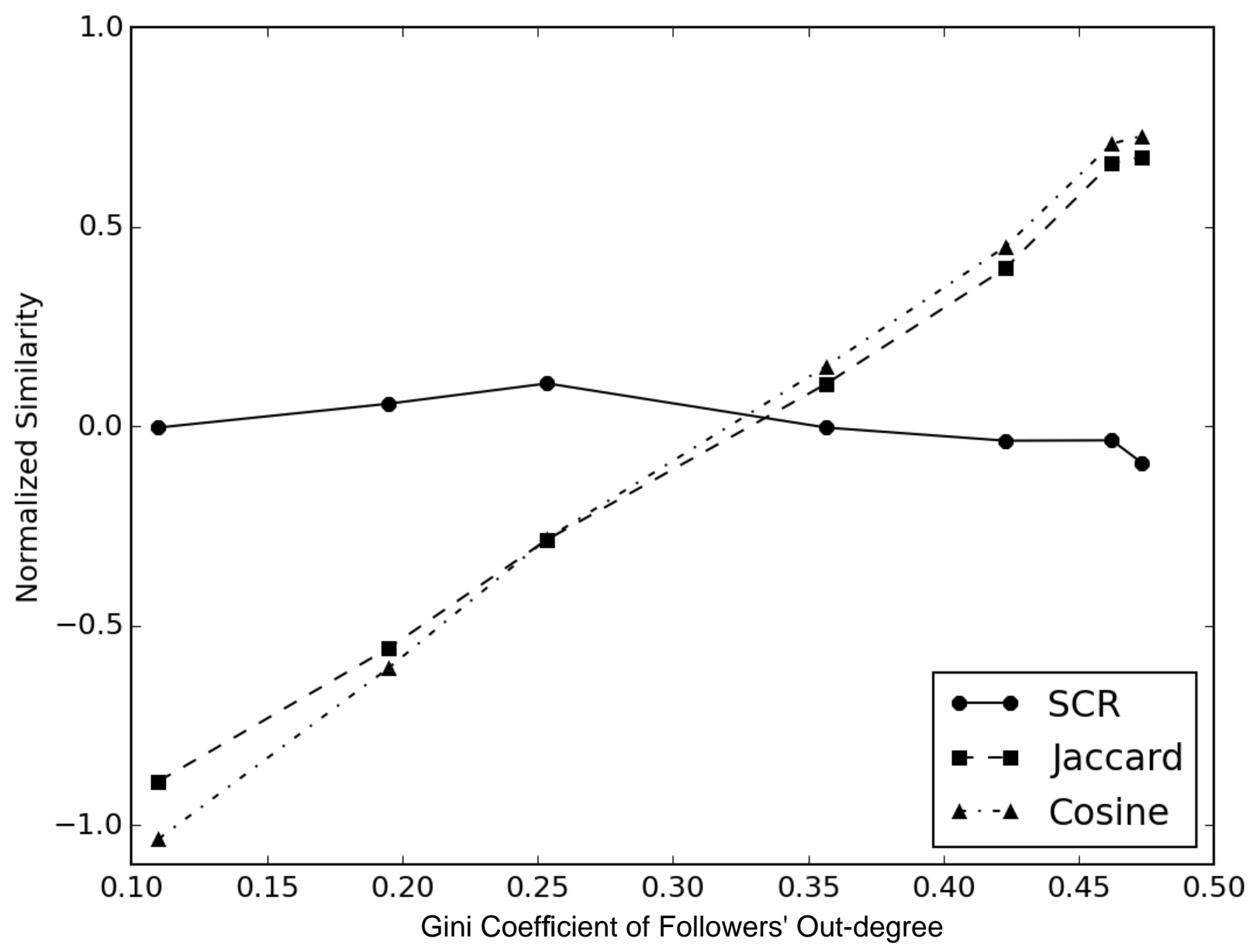


Figure 4. U.S. Congress members by party affiliation as Republican (red), Democrat (blue), and Independent (green). Node size indicates follower count and pairwise proximity corresponds to SCR.

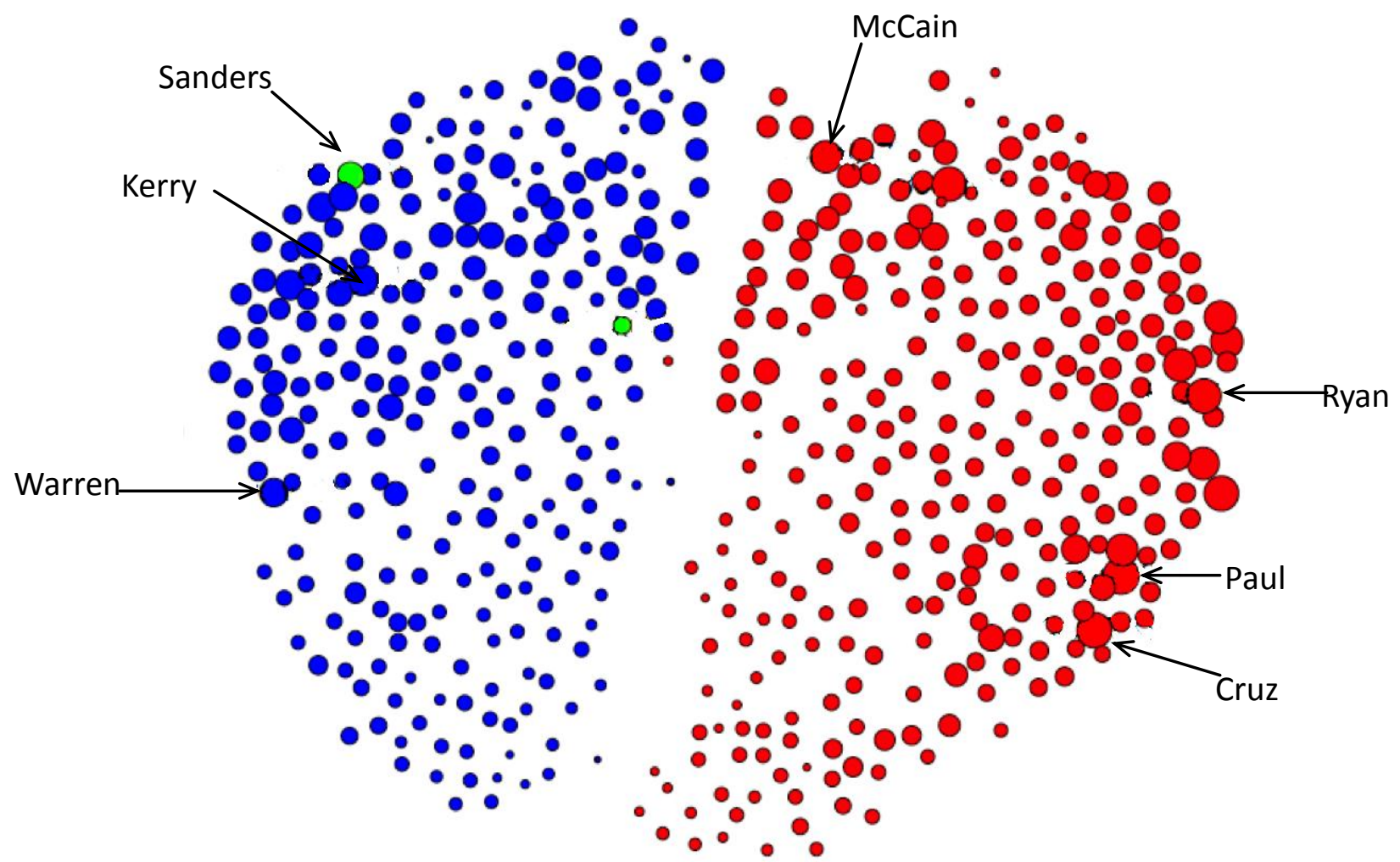


Figure 5. Musical artists by genre. Node size indicates follower count and pairwise proximity corresponds to SCR.
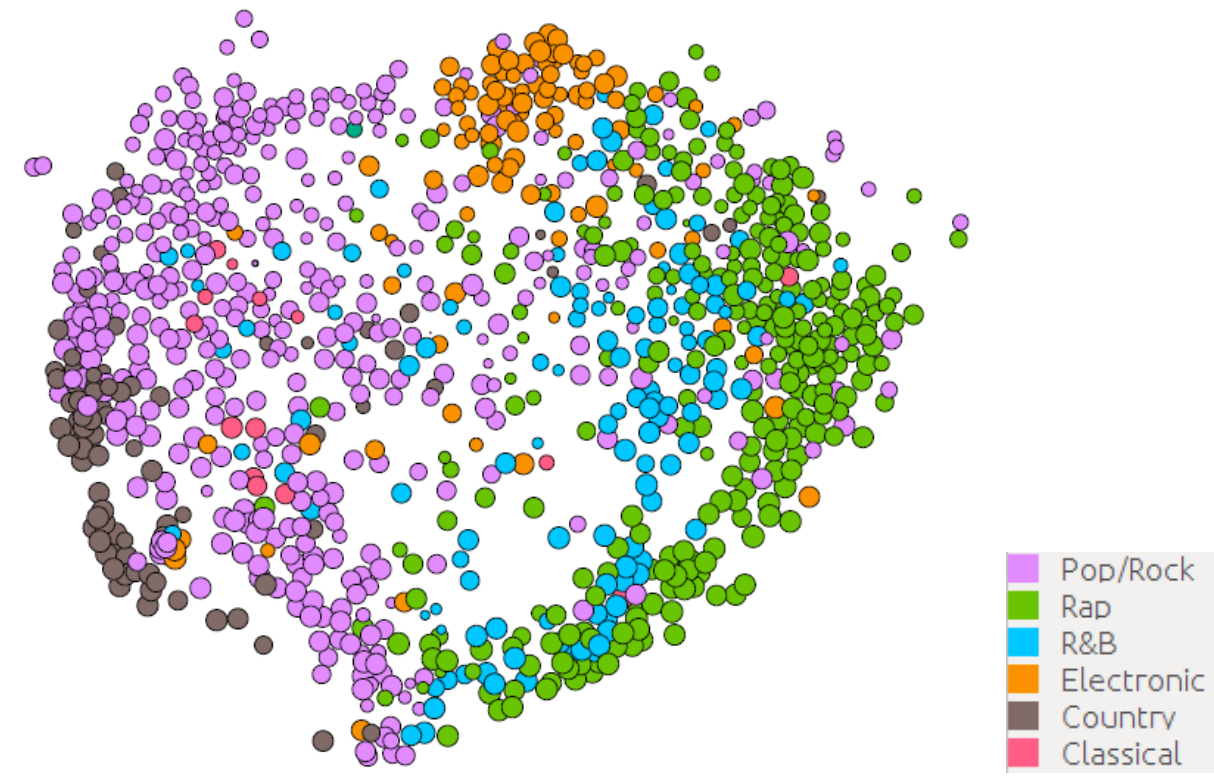
Figure 6. Professional sports teams by league. Node size indicates follower count and pairwise proximity corresponds to SCR.

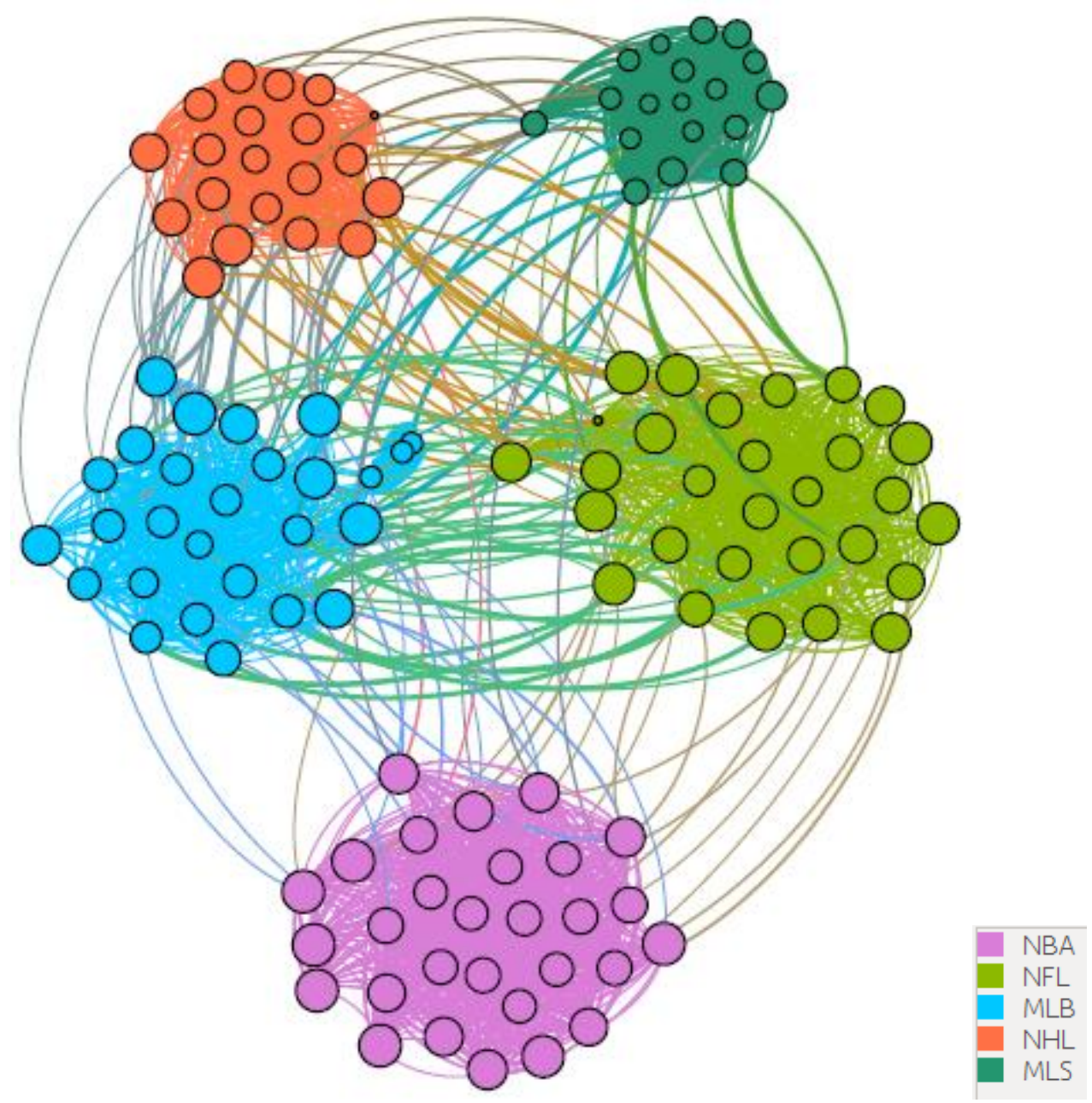

